\title{
Hybrid Quantum Processors: Molecular Ensembles as Quantum Memory for Solid State Circuits
}

\section{Citation}

Rabl, P., D. DeMille, J. M. Doyle, M. D. Lukin, R. J. Schoelkopf, and P. Zoller. 2006. "Hybrid Quantum Processors: Molecular Ensembles as Quantum Memory for Solid State Circuits." Physical Review Letters 97 (3) (July 21). doi:10.1103/physrevlett.97.033003.

\section{Published Version}

doi:10.1103/PhysRevLett.97.033003

\section{Permanent link}

http://nrs.harvard.edu/urn-3:HUL.InstRepos:27867252

\section{Terms of Use}

This article was downloaded from Harvard University's DASH repository, and is made available under the terms and conditions applicable to Other Posted Material, as set forth at http:// nrs.harvard.edu/urn-3:HUL.InstRepos:dash.current.terms-of-use\#LAA

\section{Share Your Story}

The Harvard community has made this article openly available.

Please share how this access benefits you. Submit a story.

\section{Accessibility}




\title{
Hybrid Quantum Processors: Molecular Ensembles as Quantum Memory for Solid State Circuits
}

\author{
P. Rabl, ${ }^{1}$ D. DeMille, ${ }^{2}$ J. M. Doyle, ${ }^{3}$ M. D. Lukin, ${ }^{3}$ R. J. Schoelkopf, ${ }^{2,4}$ and P. Zoller ${ }^{1}$ \\ ${ }^{1}$ Institute for Theoretical Physics, University of Innsbruck, and Institute for Quantum Optics \\ and Quantum Information of the Austrian Academy of Sciences, A-6020 Innsbruck, Austria \\ ${ }^{2}$ Department of Physics, Yale University, New Haven, Connecticut 06520, USA \\ ${ }^{3}$ Department of Physics, Harvard University, Cambridge, Massachusetts 02138, USA \\ ${ }^{4}$ Department of Applied Physics, Yale University, New Haven, Connecticut 06520, USA
}

(Received 19 April 2006; published 21 July 2006)

\begin{abstract}
We investigate a hybrid quantum circuit where ensembles of cold polar molecules serve as long-lived quantum memories and optical interfaces for solid state quantum processors. The quantum memory realized by collective spin states (ensemble qubit) is coupled to a high- $Q$ stripline cavity via microwave Raman processes. We show that, for convenient trap-surface distances of a few $\mu \mathrm{m}$, strong coupling between the cavity and ensemble qubit can be achieved. We discuss basic quantum information protocols, including a swap from the cavity photon bus to the molecular quantum memory, and a deterministic two qubit gate. Finally, we investigate coherence properties of molecular ensemble quantum bits.
\end{abstract}

DOI: 10.1103/PhysRevLett.97.033003

During the last few years we have witnessed remarkable progress towards the realization of quantum information processing in various physical systems. Highlights include quantum optical systems of trapped atoms and ions [1], and cavity QED [2], as well as solid state systems including Cooper pair boxes (CPB) [3,4] and quantum dots [5]. In particular, the strong coupling regime of circuit CQED [6] was realized using a $\mathrm{CPB}$ strongly coupled to a stripline cavity. In the light of these developments it is timely to investigate hybrid devices with the goal of combining the advantages of various implementations, i.e., to build interfaces between, for example, a quantum optics and solid state qubit with compatible experimental setups [7]. Such interfaces are particularly important in applications where long-term quantum memories or optical interconnects are required $[8,9]$.

In this Letter we study such a scenario by coupling a stripline cavity to a cloud of cold polar molecules [10]. The cavity may be part of a solid state quantum processor involving CPB as charge qubits [3] and microwave photons as a quantum data bus, while the molecular ensemble serves as a quantum memory with a long coherence time. The condition of strong coupling between the cavity and the molecular cloud is achieved via the (large) electric dipole moments of polar molecules for rotational excitations in the electronic and vibrational ground state, which are in the tens of $\mathrm{GHz}$ regime, and thus provide an ideal match for resonance frequencies of stripline cavities. By adopting a molecular ensemble instead of a single polar molecule [11], we benefit from the enhancement of the coherent coupling $g \sqrt{N}$ with the number of molecules $N$ and $g$ the single molecule vacuum Rabi frequency. This strong coupling between the molecular ensembles and the circuit CQED system also opens the possibility of a solid state based readout of molecular qubits. In addition, qubits stored in the molecular ensemble can be converted to "flying" optical qubits, using techniques demonstrated
PACS numbers: 33.80.Ps, 03.67.Lx, 42.50.Dv, 85.25.Cp

for atomic ensembles [12]. This provides a natural interface between mesoscopic quantum circuits and optical quantum communication.

Let us consider the setup of Fig. 1, where two molecular ensembles are coupled to a superconducting cavity. The cavity is assumed to be strongly coupled to a CBP representing a circuit CQED system, as realized in recent experiments at Yale [6]. As discussed in detail below, molecular spectroscopy allows us to identify long-lived states, for example, in the form of a spin qubit $|0\rangle,|1\rangle$ in the ground rotational manifold. Starting with a cloud of $N$ molecules prepared in $|0\rangle_{m} \equiv\left|0_{1} 0_{2} \ldots 0_{N}\right\rangle$ coupling to a microwave or cavity field leads to excitations in the form of symmetric Dicke states, $|1\rangle_{m} \equiv 1 / \sqrt{N} \sum_{i}\left|0_{1} \ldots 1_{i} \ldots 0_{N}\right\rangle \equiv$ $m^{\dagger}|0\rangle_{m}$, etc. For weak excitation the operator $m$ obeys approximate harmonic oscillator commutation relations $\left[m, m^{\dagger}\right] \approx 1$, and the ensemble excitations are conveniently described as a set of harmonic oscillator states
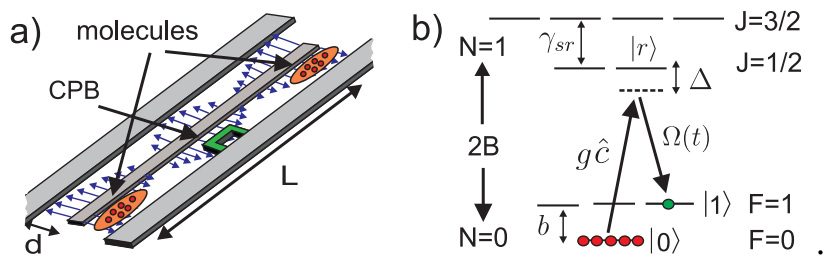

FIG. 1 (color online). (a) Ensembles of polar molecules and a CPB are coupled via the quantized field of a stripline cavity (see text for more details). (b) Rotational excitation spectrum of molecules with a ${ }^{2} \Sigma_{1 / 2}$ ground state where we plot excited states according to the Hamiltonian $H=H_{R}+H_{\mathrm{SR}}$. For nonzero nuclear spin $\vec{I}$ (here $I=1 / 2$ ) the hyperfine interaction leads to an additional splitting ( $b \sim 2 \pi \times 100 \mathrm{MHz}$ ) into eigenstates of $\vec{F}=\vec{J}+\vec{I}$ (hyperfine splitting for the excite states is not shown). Two qubit states in the $N=0$ manifold, $|0\rangle,|1\rangle$ are coupled by a Raman process involving a single cavity photon and an external microwave field, $\Omega(t)$. 
$|0\rangle_{m},|1\rangle_{m} \equiv m^{\dagger}|0\rangle_{m}$, etc. Our goal below is to use the lowest two of these states as ensemble qubits, which can be manipulated by coupling them to the superconducting cavity and a CPB.

The dynamics of the coupled system [Fig. 1(a)] can be described in terms of a Hamiltonian $H_{\text {sys }}=H_{C}+H_{M}+$ $H_{\mathrm{CM}}$, which is the sum of a Jaynes-Cummings type Hamiltonian for the circuit CQED system $H_{C}$, a Hamiltonian for the (spin) excitations of the molecular ensembles $H_{M}$, and the coupling of the molecules to the cavity $H_{\mathrm{CM}}$. In a frame rotating with the cavity frequency $\omega_{c}$ the CQED Hamiltonian has the form

$$
H_{C}=-\delta_{c}(t)|e\rangle\langle e|+g_{c}\left(|e\rangle\langle g|\hat{c}+| g\rangle\langle e| \hat{c}^{\dagger}\right) .
$$

Here $|g\rangle$ and $|e\rangle$ denote the ground and the first excited eigenstate of the $\mathrm{CPB}$ at the charge degeneracy point representing a charge qubit with a (tunable) transition frequency $\omega_{c q}(t)$ and a detuning from the cavity $\delta_{c}(t)=$ $\omega_{c}-\omega_{\mathrm{cq}}(t)$. The operator $\hat{c}\left(\hat{c}^{\dagger}\right)$ is the cavity annihilation (creation) operator for microwave photons. The Hamiltonian describing the internal excitations of the molecular ensembles $i=1,2$ and the coupling of ensemble states to the stripline cavity takes the form

$$
H_{M}+H_{\mathrm{CM}}=-\sum_{i} \delta_{m, i}(t) m_{i}^{\dagger} m_{i}+\sum_{i} g_{m, i}(t) m_{i}^{\dagger} \hat{c}+\text { H.c. }
$$

Before we enter the details of the derivation of $H_{M}$ and $H_{\mathrm{CM}}$ we note the basic structure of the Hamiltonian $H_{\text {sys }}$. The ensemble excitations and the cavity represent a system of coupled harmonic oscillators interacting with a twolevel system (CPB) with controllable coefficients. In general, this provides the basic ingredients for (i) swap operations between charge, cavity, and ensemble qubits, (ii) rotations of a single ensemble qubit via the charge qubit, and (iii) 2-qubit entanglement operations between two ensemble qubits, where the charge qubit plays the role of a nonlinearity. For example, the CPB can act as a "single photon source"; i.e., we generate a superposition state of the charge qubit, which by an appropriate control sequence can be swapped over to the cavity, and is finally stored in one of the molecular ensembles, and vice versa: $(\alpha|g\rangle+$ $\beta|e\rangle)|0\rangle_{c}|0\rangle_{m} \rightarrow|g\rangle\left(\alpha|0\rangle_{c}+\beta|1\rangle_{c}\right)|0\rangle_{m} \rightarrow|g\rangle|0\rangle_{c}\left(\alpha|0\rangle_{m}+\right.$ $\left.\beta|1\rangle_{m}\right)$.

The above discussion has ignored various sources of decoherence. In the Yale experiment [6], the circuit CQED system realizes the strong coupling regime with vacuum Rabi frequency $g_{c} \leqslant 2 \pi \times 50 \mathrm{MHz}$. The decoherence of the charge qubit is dominated by the dephasing rate $T_{2}^{-1} \approx 2 \pi \times 0.5 \mathrm{MHz}$, while the photon loss rate is $\kappa / 2 \pi=1$ to $0.01 \mathrm{MHz}$; i.e., the charge qubit is the dominant source of decoherence. Below we show that for a cloud of $N \approx 10^{4}$ to $10^{6}$ molecules, trapped $10 \mu \mathrm{m}$ above the stripline cavity, one can reach the regime of strong cavity-ensemble coupling $g_{m} / 2 \pi \approx 1-10 \mathrm{MHz}$, which should be compared with the expected collisional dephasing rates of a few hundred $\mathrm{Hz}$.
Figure 1(b) shows the rotational spectrum of $\mathrm{CaF}$, which provides an example for spectra of alkaline-earth monohalogenides with a ${ }^{2} \Sigma_{1 / 2}$ ground state corresponding to a single electron outside a closed shell. The spectrum consists of rotational eigenstates, described by a rigid rotor Hamiltonian $H_{R}=B \vec{N}^{2}$ with $B \sim 2 \pi \times 10 \mathrm{GHz}$ the rotational constant, and $\vec{N}$ the angular momentum of the nuclei. The unpaired spin is coupled to the molecule rotation according to $H_{\mathrm{SR}}=\gamma_{\mathrm{sr}} \vec{S} \vec{N}$ with $\gamma_{\mathrm{sr}} \sim 2 \pi \times 40 \mathrm{MHz}$ and $\vec{S}$ the electron spin $(S=1 / 2)$. Coupled eigenstates are denoted by $\left|N, S, J ; M_{J}\right\rangle$ with $\vec{J}=\vec{N}+\vec{S}$. As seen from Fig. 1, there is a spin rotation splitting ( $\rho$ doubling) for rotationally excited states. In addition, there can be hyperfine interactions, which, in particular, lead to a splitting of the ground state $N=0$, as in the case of $\mathrm{CaF}$ with a nuclear spin $I=1 / 2$ which are coupled with $J=1 / 2$ to $F=0$ and 1 states. In the following, we denote by $|0\rangle,|1\rangle$ a pair of states in the rotational ground state manifold to provide our spin qubit. Compared to qubits stored in the rotational degrees of freedom this choice of states avoids unfavorable $N=1 \rightarrow N=0$ collisions while $|0\rangle$ and $|1\rangle$ can still be coupled efficiently by a Raman process.

The cavity mode and microwave fields of appropriate frequency and polarization couple rotational ground states to excited states with electric dipole matrix elements $\mu$ $(\sim 5 \mathrm{D})$. Two microwave driving fields provide an effective coupling Hamiltonian $\frac{1}{2} \Omega_{\text {eff }}(t)|0\rangle\langle 1|+$ H.c. to rotate the single molecule spin qubit, where $\Omega_{\text {eff }}=\Omega_{1} \Omega_{2} / 2 \Delta$, with $\Omega_{1,2}$ the Rabi frequencies and $\Delta$ the detuning from the excited state $|r\rangle,\left(\Delta \geqslant \Omega_{1,2}\right)$. By similar arguments the coupling to the cavity has the form $g_{\text {eff }}(t)|1\rangle\langle 0| \hat{c}+$ H.c. with $g_{\text {eff }}(t) \equiv g \Omega(t) / 2 \Delta$, where $g=\mu \mathcal{E}_{c}$ is the vacuum Rabi frequency, and $\mathcal{E}_{c} \approx \sqrt{\hbar \omega_{c} / 2 \pi \epsilon_{0} d^{2} L}$ is the electric field per photon for a cavity length $L$ and typical electrode distance $d$. Typical values are $g / 2 \pi \sim 5-10 \mathrm{kHz}$ for $\mu \approx$ $5 \mathrm{D}$ and $d \approx 10 \mu \mathrm{m}$. The distance $d$ is also an estimate of the trapping distance of the molecular cloud from the cavity, which is well in the limit where standard trapping techniques work reliably and surface effects are negligible. Rewriting $H_{\mathrm{CM}}$ in terms of the collective operator $m$ we obtain an effective cavity-ensemble coupling $g_{m}(t)=$ $\sqrt{N} g_{\text {eff }}(t)$. Because of the large wavelength $\lambda_{c} \approx 1.5 \mathrm{~cm}$ a trap volume of $V \leq d \times d \times \lambda_{c} / 10$ contains $N=$ $10^{4}-10^{6}$ molecules for gas densities of $n \sim 10^{12} \mathrm{~cm}^{-3}$ resulting in a coupling strength of $g_{m} / 2 \pi \sim 1-10 \mathrm{MHz}$. The parameters $\delta_{m, i}(t)$ are Raman detunings, which can be controlled independently, e.g., by applying local magnetic and/or electric fields. Thus we obtain the Hamiltonian $H_{M}+H_{\mathrm{CM}}$, which allows a SWAP of a cavity and an ensemble state, for example, by an adiabatic sweep of $\delta_{m}$ across the resonance. This corresponds to a read or write operation $\rho_{c} \otimes|0\rangle_{m}\langle 0|\longleftrightarrow| 0\rangle_{c}\langle 0| \otimes \rho_{m}$ with $\rho_{c}$ an arbitrary density operator of the microwave field in the cavity, and $\rho_{m}$ the identical state stored in ensemble excitations.

The CPB provides a nonlinear element in the Hamiltonian $H_{\text {sys. }}$. This allows first of all single qubit op- 
erations of ensemble qubits, e.g., by combining a swap operation with single qubit rotations of the charge qubit, and second, deterministic entanglement operations of qubits stored in two molecular ensembles. An example of such a protocol, which uses the $\mathrm{CPB}$ as a nonlinear phase shifter, is given as follows. We assume that the system is initially prepared in the state $|\psi\rangle_{t=0}=|g\rangle|0\rangle_{c}|\psi\rangle_{m}$ with the charge qubit far detuned from the cavity resonance, $\left|\delta_{c}(0)\right| \gg g_{c}$, and the two ensemble qubits in an arbitrary state $|\psi\rangle_{m}$ spanned by the basis $\left|\epsilon_{1} \epsilon_{2}\right\rangle_{m}, \epsilon_{i}=0,1$. In a first step, in analogy to the single qubit swap, the state $|\psi\rangle_{m}$ is (partially) transferred to the cavity. Assuming symmetric conditions, $g_{m, i}=g_{m}$ and $\delta_{m, i}(t)=\delta_{m}(t)$, it is convenient to rewrite the ensemble state in terms of the (anti)symmetric operators $m_{s / a}=\left(m_{1} \pm m_{2}\right) / \sqrt{2}$ acting on $|00\rangle_{m}$. An adiabatic sweep of the Raman detunings then realizes the swap operation $m_{s}^{\dagger}|00\rangle_{m}|0\rangle_{c} \rightarrow|00\rangle_{m}|1\rangle_{c}$ and $\left(m_{s}^{\dagger}\right)^{2}|00\rangle_{m}|0\rangle_{c} \rightarrow$ $\sqrt{2}|00\rangle_{m}|2\rangle_{c}$ while the states $|00\rangle_{m}|0\rangle_{c}, m_{a}^{\dagger}|00\rangle_{m}|0\rangle_{c}$, and $\left(m_{a}^{\dagger}\right)^{2}|00\rangle_{m}|0\rangle_{c}$ remain unaffected. In a second step, the charge qubit is adiabatically tuned close to resonance for a time $T,\left|\delta_{c}(T / 2)\right| \lesssim g_{c}$. During this pulse the nonvacuum states acquire a nonlinear dynamical phase, $|n\rangle_{c} \rightarrow$ $e^{i \phi_{n}}|n\rangle_{c}$, with $\phi_{n}=-\int_{0}^{T} d t^{\prime}\left(\delta_{c}\left(t^{\prime}\right)+\sqrt{\delta_{c}^{2}\left(t^{\prime}\right)+n 4 g_{c}^{2}}\right) / 2$. The pulse form $\delta_{c}(t)$ and the length $T$ are chosen such that $\phi_{1} \simeq \pi / 2$ and $\phi_{2}=2 \pi n$ (see, e.g., Fig. 2). The second condition ensures that after writing the cavity state back into the ensembles states, i.e., reversing step one, the ensemble states $|20\rangle_{m}$ and $|02\rangle_{m}$ remain unpopulated. The total gate sequence corresponds to a $\sqrt{\mathrm{SWAP}}-$ like gate for two ensembles qubits, with $|00\rangle_{m} \rightarrow|00\rangle_{m},|10\rangle_{m} \rightarrow$ $e^{i \pi / 4}\left(|10\rangle_{m}+i|01\rangle_{m}\right) / \sqrt{2}, \quad|01\rangle_{m} \rightarrow e^{i \pi / 4}\left(i|10\rangle_{m}+|01\rangle_{m}\right) /$ $\sqrt{2}$, and $|11\rangle_{m} \rightarrow|11\rangle_{m}$. A numerical simulation of this gate sequence based on a master equation treatment of the dissipative terms [6] shows that the gate fidelity is only limited by $\left(g_{c} T_{2}\right)^{-1}$; i.e., the decoherence of the $\mathrm{CPB}$ during the time it is tuned close to resonance (see Fig. 2 for more details).

We now turn to an analysis of decoherence in the molecular ensemble. In particular, collisional dephasing of the
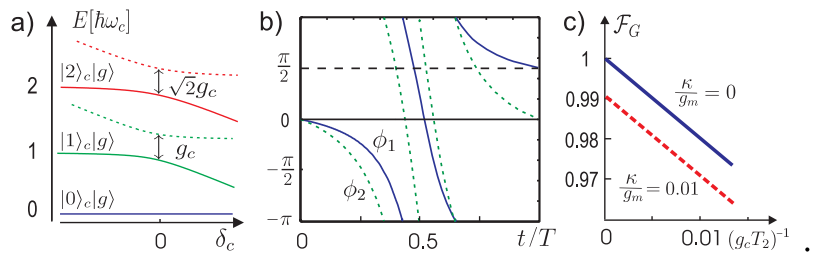

FIG. 2 (color online). (a) Adiabatic energies levels for the states $|n\rangle_{c}|g\rangle$ as a function of the charge qubit detuning $\delta_{c}$. (b) Evolution of the dynamical phases $\phi_{1}$ (solid line) and $\phi_{2}$ (dashed line) for a pulse $\delta_{c}(t)=-\delta_{0}(2 t / T-1)^{2}-\delta_{1}$, $\delta_{0} / g_{c}=30, \delta_{1} / g_{c}=0.44$, and $T=44.79 / g_{c}$. For the same pulse $\delta_{c}(t)$ the resulting fidelity of the total gate sequence, $\mathcal{F}_{G}$ (averaged over all initial states $|\psi\rangle_{m}$ ), is plotted in (c) for different values of the charge qubit dephasing rate, $T_{2}^{-1}$, and the cavity loss rate $\kappa$. ensemble qubit, and a spatial variation of the cavitymolecule coupling $g_{\text {eff }}(x)$ in combination with the thermal motion of the molecules in the trap, contribute to a finite decoherence time of the molecular quantum memory, and result in imperfections during gate operations.

An operational definition of the decoherence time of the ensemble qubit can be given in terms of an (idealized) experiment. A cavity qubit $\rho_{c}(t=0)=|\psi\rangle_{c}\langle\psi|$ with $|\psi\rangle_{c}=\alpha|0\rangle_{c}+\beta|1\rangle_{c}$ is written at time $t=0$ to the molecular memory with all molecules initialized in the state $|0\rangle$ in a (perfect) swap operation, kept in storage for a time interval $\tau$, undergoing dephasing collisions. At $t=\tau$, the qubit is transferred back to the cavity mode, resulting in a reduced density matrix $\rho_{c}(\tau)$ of the cavity with a fidelity $F_{\tau}=\min _{\psi_{c} c}\left\langle\psi\left|\rho_{c}(\tau)\right| \psi\right\rangle_{c}$, with the decoherence time of the ensemble memory identified as the decay time of the fidelity. The analysis of this process resembles the discussions of clock shifts, and, in particular, studies of collisional dephasing of spins in thermal and quantum degenerate atomic clouds in a Ramsey interferometry setup. A formal theoretical description of these phenomena is provided by quantum kinetic theory [13].

We consider a cloud of molecules in the lowest rotational state with external degrees of freedom cooled to a temperature $T_{m} \lesssim 1 \mathrm{mK}[10,11]$ and qubits stored in spin or hyperfine states (Fig. 1). Molecules in the rotational ground state are trapped magnetically [14] or by a (spin independent) electric rf trap [15]. In a magnetic trap two molecules interact asymptotically according to a $V(r) \simeq$ $-C_{6} / r^{6}$ potential with $C_{6}=\left(\mu^{2} / 4 \pi \epsilon_{0}\right)^{2} / 6 B$. The effective range of this potential is given by $R_{*}=\sqrt[4]{m C_{6} / \hbar^{2}}$, which provides an estimate for the $s$-wave scattering length $\bar{a}$. For example, for $\mathrm{CaCl}$ (which has two magnetically trapped hyperfine states) we obtain $R_{*} \approx 780 a_{B}$ which is a few times the typical scattering length encountered for alkali atoms. $S$-wave scattering dominates for temperatures $T_{m} \lesssim T_{*} \approx 1 \mu \mathrm{K}$ where the thermal energy is below the centrifugal barrier for higher angular momenta, leading to an estimate for the collision rate $\gamma_{\mathrm{col}}=8 \pi \bar{a}^{2} n \bar{v} \approx 2 \pi \times$ $150 \mathrm{~Hz}$ for $n=10^{12} \mathrm{~cm}^{-3}$ and $\bar{v}$ the relative thermal velocity. For temperatures $T_{m} \gg T_{*}$ higher partial waves contribute, and an estimate of the cross section based on the unitarity limit gives $\gamma_{\text {col }} \leqslant 2 \pi \times 700 \mathrm{~Hz}$ for $T_{m}=$ $1 \mathrm{mK}$. In electric traps the induced dipole moment $\mu_{\text {ind }}$ leads to a $V(r) \sim \mu_{\text {ind }}^{2} / r^{3}$ dependence of the asymptotic interaction. Although this long-range behavior significantly changes the low temperature scattering $\left(T_{m}<\right.$ $1 \mu \mathrm{K})$ the estimate based on the unitarity limit at $T_{m} \approx$ $1 \mathrm{mK}$ still provides a valid bound for scattering rates for $\mu_{\text {ind }}<1 \mathrm{D}$.

We have calculated the decoherence time of an ensemble qubit corresponding to collisional dephasing using quantum kinetic theory [16]. Dephasing of the qubit coherence $\left(\rho_{c}\right)_{10}(\tau)=\exp \left(-\gamma_{10} \tau / 2\right)\left(\rho_{c}\right)_{10}(0)$ is associated with spin dependent collisions between the states $|0\rangle$ and $|1\rangle$, which gives a contribution 


$$
\begin{aligned}
\gamma_{10}= & \frac{2 \hbar^{3} n}{m^{2}} \int \prod_{i=1 . .4} d^{3} k_{i} \delta(\Delta E(\mathbf{K})) \delta(\mathbf{K}) P\left(\vec{k}_{1}\right) P\left(\vec{k}_{2}\right) \\
& \times\left(\left|f_{00}^{e}(\mathbf{K})-f_{01}^{e}(\mathbf{K})\right|^{2}+\left|f_{00}^{\text {in }}(\mathbf{K})\right|^{2}+\left|f_{01}^{\text {in }}(\mathbf{K})\right|^{2}\right) .
\end{aligned}
$$

It depends on the difference between $f_{00}^{e}$ and $f_{01}^{e}$, the elastic scattering amplitudes for the internal states $|00\rangle$ and $(|10\rangle+|01\rangle) / \sqrt{2}$ averaged over the thermal distributions $P(\vec{k})$ in the scattering process between momenta $\mathbf{K}=$ $\left(\vec{k}_{3}, \vec{k}_{4} \leftarrow \vec{k}_{1}, \vec{k}_{2}\right)$, and $\delta$ functions accounting for energy and momentum conservation in the collision. In addition, there may be contributions from inelastic collisions, $f_{00}^{\text {in }}$ and $f_{01}^{\text {in }}$, which scatter molecules outside the $|0\rangle,|1\rangle$ subspace. While accurate scattering amplitudes for molecular collisions may not be available at present, we can estimate these contributions in certain limits. For $s$-wave scattering the above expression simplifies to $\gamma_{10}=8 \pi\left(a_{00}-\right.$ $\left.a_{01}\right)^{2} n \bar{v}$ with $a_{00}$ and $a_{01}$ scattering lengths. If we assume that the scattering length is dominated by a spin exchange potential, the scattering is characterized by a singlet $\left(a_{S}\right)$ and triplet scattering length $\left(a_{T}\right)$. In the simple case of a pure spin qubit $\{|0\rangle,|1\rangle\} \equiv\left\{\left|S=1 / 2, m_{s}= \pm 1 / 2\right\rangle\right\}$ we find $a_{00}=a_{01}=a_{T}$, and the dephasing rate is determined by nonvanishing contributions arising from magnetic dipole and spin rotation coupling, which are expected to be much smaller. In a similar way, in the presence of hyperfine interactions we can form a qubit $|0\rangle=\mid F=I+$ $\left.1 / 2, M_{F}=F\right\rangle$ and $|1\rangle=\left|F^{\prime}=I-1 / 2, M_{F}=F^{\prime}\right\rangle$, where again $|00\rangle$ and $|01\rangle+|10\rangle$ contain no spin singlet contribution and the leading dephasing term vanishes. In the worst case the decoherence rate is bounded by the single molecule collision rate $\gamma_{10} \approx \gamma_{\text {col }}$ which has been estimated above.

Spatial variations of the effective single moleculecavity coupling, $g_{\text {eff }}(x)$, result in a dephasing of the qubit during a single swap gate and an incomplete recovery of the state after a redistribution of the molecules between two successive write or read operations. The inhomogeneity in the coupling arises from the variation of the cavity mode function on a scale of the electrode distance, $g(x) \approx g(1-\alpha x / d)$, with $\alpha$ a numerical constant, and a position dependence of the detuning $\Delta(x) \approx \Delta-$ $m \delta \omega^{2} x^{2} /(2 \hbar)$. Here $\delta \omega^{2}=\omega_{t}^{2}-\omega_{r}^{2}$ accounts for a difference in the trapping potentials for the qubit states $\left(\omega_{t}\right)$ and the excited state $|r\rangle\left(\omega_{r}\right)$. For an optimal detuning $\Delta_{*} \simeq$ $\sqrt[3]{3 g^{2} N\left(k_{b} T_{m} \delta \omega^{2}\right)^{2} / \kappa \omega_{t}^{4} \hbar^{2}}$ the inhomogeneous coupling results in a total gate error of $\epsilon \approx \alpha^{2}\left(k_{b} T_{m} / m \omega_{t}^{2} d^{2}\right)+$ $\left(k_{b} T_{m} \delta \omega^{2} \kappa / \hbar g^{2} N \omega_{t}^{2}\right)^{2 / 3} \quad[16]$. For $g \sqrt{N} \sim 2 \pi \times$ $10 \mathrm{MHz}, \kappa \sim 2 \pi \times 10 \mathrm{kHz}$ and at $T_{m}=1 \mathrm{mK}$ gate fidelities of $\mathcal{F}>0.99$ require trap frequencies of $\omega_{t} \sim 2 \pi \times$ $50 \mathrm{kHz}$ and a similar trapping potential for the state $|r\rangle$ $\left(\delta \omega^{2} \sim 0.1 \omega_{t}^{2}\right)$. Lower temperatures and an optimized cavity or trap design, e.g., $\alpha, \delta \omega^{2} \rightarrow 0$, lead to a further significant reduction of gate errors.
In conclusion, ensembles of cold polar molecules represent a good quantum memory that can be strongly coupled to stripline cavities with a long lifetime, limited essentially only by collisional dephasing. We note that these dephasing channels are virtually eliminated, if the ensemble is prepared in a crystalline phase of dipolar gases with dipole moments induced and aligned by a dc electric field under 2D trapping conditions [16]. The present work opens an exciting avenue towards long-lived molecular quantum memories for solid state quantum processors.

Work at Innsbruck is supported by the Austrian Science Foundation, European Networks, and the Institute for Quantum Information. P. R. thanks the Harvard Physics Department and ITAMP for hospitality. Work at Harvard is supported by NSF, Harvard-MIT CUA, and Packard and Sloan Foundations. Work at Yale is supported by NSF Grant No. DMR0325580, the W. M. Keck Foundation, and the Army Research Office.

[1] D. Leibfried et al., Nature (London) 438, 639 (2005); H. Häffner et al., Nature (London) 438, 643 (2005).

[2] S. Numann et al., Nature Phys. 1, 122 (2005); K. M. Birnbaum et al., Nature (London) 436, 87 (2005).

[3] Yu. Makhlin et al., Rev. Mod. Phys. 73, 357 (2001).

[4] D. Vion, et al., Science 296, 886 (2002); I. Chiorescu et al., Nature (London) 431, 159 (2004).

[5] J.R. Petta et al., Science 309, 2180 (2005); F. H. L. Koppens et al., Science 309, 1346 (2005).

[6] A. Wallraff et al., Nature (London) 431, 162 (2004); A. Blais et al., Phys. Rev. A 69, 062320 (2004).

[7] For early ideas involving hybrid implementations, see A. S. Sorensen et al., Phys. Rev. Lett. 92, 063601 (2004); L. Tian et al., Phys. Rev. Lett. 92, 247902 (2004).

[8] See, e.g., A. M. Steane, Quantum Inf. Comput. 2, 297 (2002).

[9] D. Bouwmeester, A. K. Ekert, and A. Zeilinger, The Physics of Quantum Information (Springer, New York, 2000).

[10] For a review, see J. Doyle et al., Eur. Phys. J. D 31, 149 (2004), and references cited within.

[11] A. Andre et al., quant-ph/0605201.

[12] M. D. Lukin, Rev. Mod. Phys. 75, 457 (2003); C. W. Chou et al., Nature (London) 438, 828 (2005); T. Chanelièrea et al., ibid. 438, 833 (2005); M. D. Eisaman et al., ibid. 438, 837 (2005); B. Julsgaard et al., ibid. 432, 482 (2004).

[13] C. W. Gardiner and P. Zoller, Phys. Rev. A 55, 2902 (1997); A. S. Bradley and C. W. Gardiner, J. Phys. B 35, 4299 (2002).

[14] Note that magnetic trapping achieved using localized magnetic fields near the nodes of the stripline resonator should not significantly degrade the quality of the superconducting cavity.

[15] J. van Veldhoven, H.L. Bethlem, and G. Meijer, Phys. Rev. Lett. 94, 083001 (2005).

[16] P. Rabl et al. (to be published); P. Xue et al. (to be published). 